\title{
Evaluation of the Effect of Single Dose of Prophylactic Ciprofloxacin Before Simple Cystourethroscopy on the Incidence of Urinary Tract Infection: A Randomized Clinical Trial
}

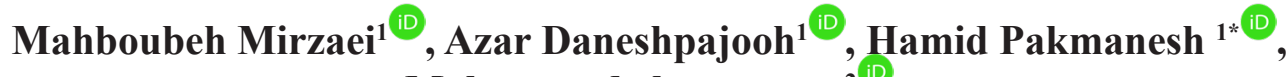 \\ Mohammad ehsan parsa ${ }^{2}$
}

1. Department of Urology, Shahid Bahonar Hospital, Kerman University of Medical Sciences (KMU), Kerman, Iran

2. Medicine Student, Kerman University of Medical Sciences (KMU), Kerman, Iran

\begin{tabular}{|c|c|}
\hline Article Information & Abstract \\
\hline Article History & \multirow{4}{*}{$\begin{array}{l}\text { Background \& Objective: The assumed necessity of antimicrobial pro- } \\
\text { phylaxis prior to simple cystoscopy is controversial. The aim of this study } \\
\text { was to determine whether antibiotic prophylaxis is required before this out- } \\
\text { patient procedure. }\end{array}$} \\
\hline Received: $\quad 2019 / 08 / 09$ & \\
\hline Accepted: $\quad 2019 / 12 / 22$ & \\
\hline Available Online: $2020 / 05 / 24$ & \\
\hline JUR 2019; 3(2):49-56 & $\begin{array}{l}\text { Methods: A total of } 162 \text { patients with mean age of } 51 \text { years old (range: } \\
\text { 12-82 years old) were recruited. They were randomized into two groups: }\end{array}$ \\
\hline DOI: 10.30699/jru.3.2.49 & $\begin{array}{l}\text { First group did not receive antibiotics ( } 81 \text { patients) and the other group re- } \\
\text { ceived prophylactic antibiotic before the operation ( } 81 \text { patients with a sin- }\end{array}$ \\
\hline $\begin{array}{l}\text { Use your device to scan } \\
\text { and read the article online }\end{array}$ & $\begin{array}{l}\text { gle dose of ciprofloxacin } 500 \mathrm{mg} \text { ). All patients underwent a diagnostic rigid } \\
\text { cystoscopy. } 48-72 \text { hours following the operation, urine cultures were taken }\end{array}$ \\
\hline 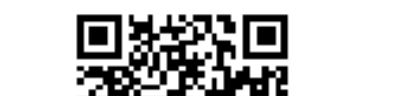 & $\begin{array}{l}\text { from all of patients. Further, patients were questioned about newly devel- } \\
\text { oped symptoms like dysuria, frequency, urgency and fever after cystoscopy. }\end{array}$ \\
\hline 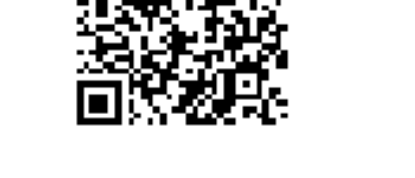 & $\begin{array}{l}\text { Results: Bacteriuria was present in } 2 / 46 \% \text { of the patients in the group } \\
\text { that received antibiotic, compared to } 4 / 93 \% \text { in the group that did not re- } \\
\text { ceive antibiotic }(P=0 / 27) \text {. Multivariate analysis in two groups showed that } \\
\text { positive bacteriuria was not associated with gender }(P=0 / 12) \text {, but there was }\end{array}$ \\
\hline Corresponding Author & $\begin{array}{l}\text { association for older than } 60 \text { years age }(P=0 / 03) \text { as well as cigarette smoking } \\
(P=0 / 04) \text {. }\end{array}$ \\
\hline $\begin{array}{l}\text { Hamid Pakmanesh } \\
\text { Assistant Professor, Depart- } \\
\text { ment of Urology, , Kerman }\end{array}$ & $\begin{array}{l}\text { Conclusions: The incidence of the urinary tract infection is not increased } \\
\text { without using prophylactic antibiotics. }\end{array}$ \\
\hline $\begin{array}{l}\text { University of Medical Sci- } \\
\text { ences (KMU), Kerman, Iran }\end{array}$ & Keywords: Rigid cystoscopy, Prophylaxis, Urinary tract infection \\
\hline $\begin{array}{l}\text { Email: } \\
\text { h_pakmanesh@kmu.ac.ir }\end{array}$ & \\
\hline
\end{tabular}

How to cite this article:

Mirzaei M, Daneshpajooh A, Pakmanesh H, Parsa M E. Evaluation of the Effect of Single Dose of Prophylactic Ciprofloxacin Before Simple Cystourethroscopy on the Incidence of Urinary Tract Infection: A Randomized Clinical Trial. J Res Urol. 2019; 3 (2) 


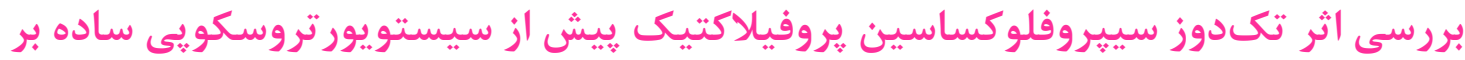

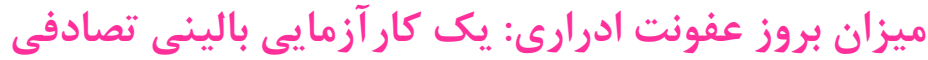

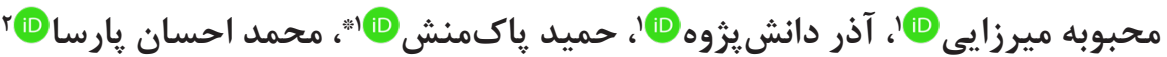

1. استاديار، گروه ارولوزى، دانشكده يزشكى، دانشكاه علوميزشكى كرمان، كرمان، ايران

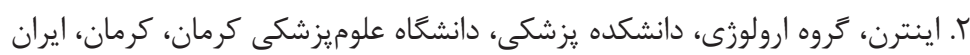

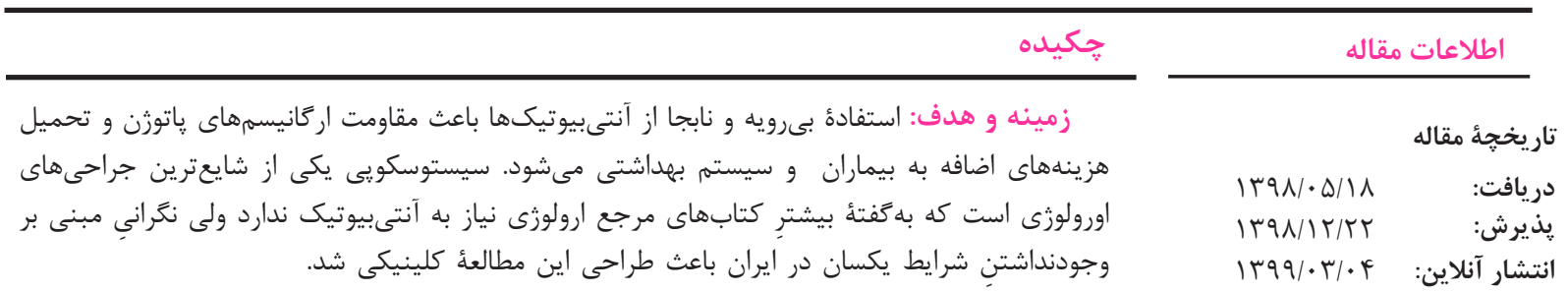

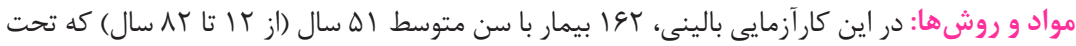

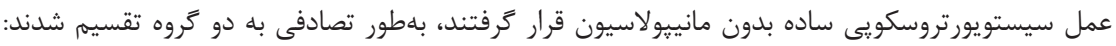

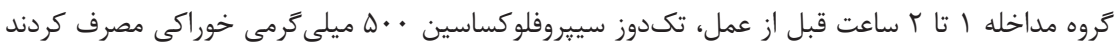

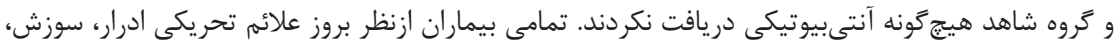

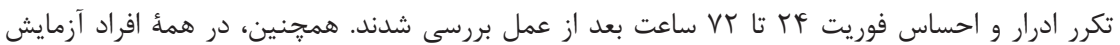

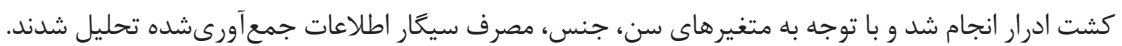

JUR 2019; 3(2):49-56

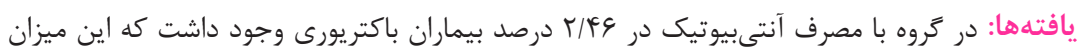

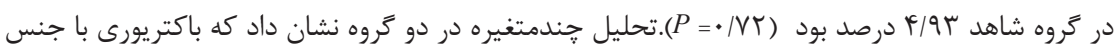

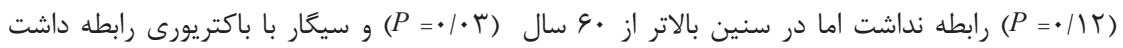

$$
(P=\cdot / \cdot \mathcal{F})
$$

نتيجهَيرى: ميزان بروز عفونت ادرارى بعد از عمل سيستويورتروسكويى ساده بدون استفاده از

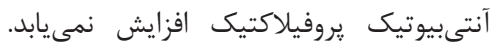

وازههاى كليدى: آنتىبيوتيك يروفيلاكسى، سيستويورتروسكويى، عفونت ادرارى
براى دانلود اين مقاله،

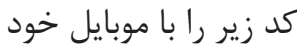

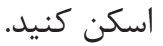

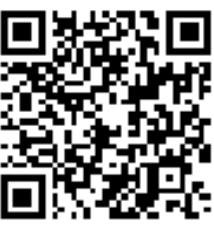

نويسنده مسئول: حميد پاكمنش، استاديار، گروه ارولوزى، دانشكده يزشكى، دانشگاه علوميزشكى كرمان، كرمان، ايران

h_pakmanesh@kmu.ac.ir 
در تمامى اعمال جراحى اورولوزى آنتىبيوتيك دريافت كنند.

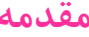

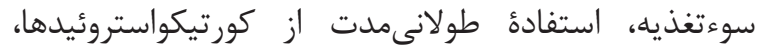

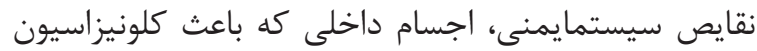

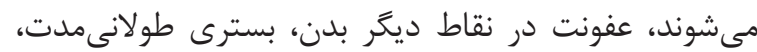

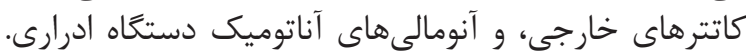

ז. فاكتورهاى مربوط به اعمال جراحى كه احتمال نفوذ

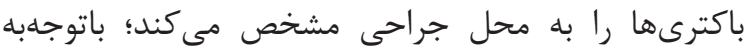

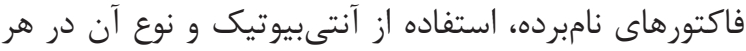

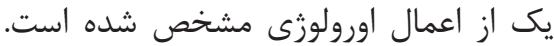

بر اين اساس افرادى كه تحت سيستويورتروسكويى ساده

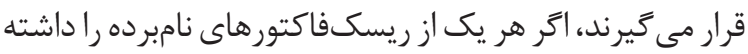

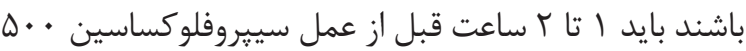

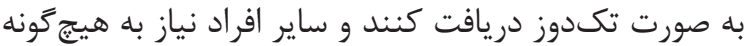

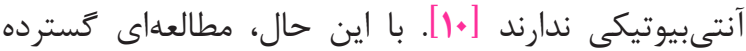

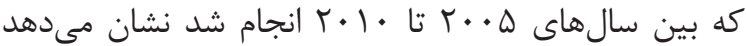

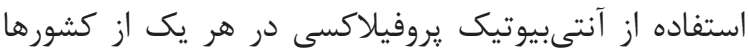

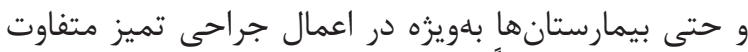

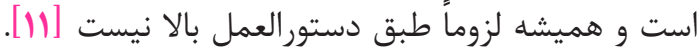
سيستوسكويى يكى از شايعترين جراحى هاى اورولوزئ است

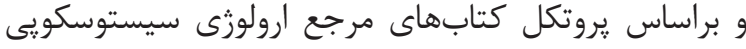

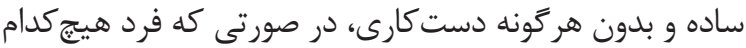

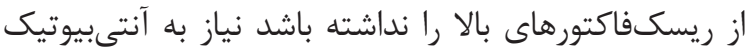

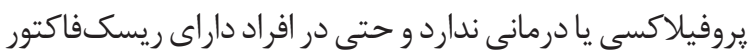

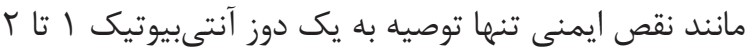

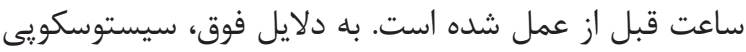

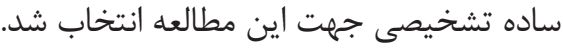

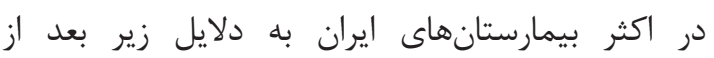

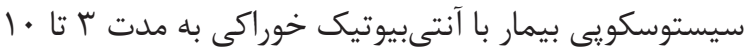

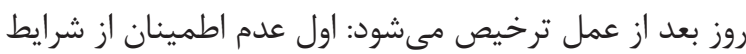

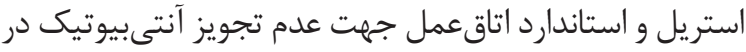

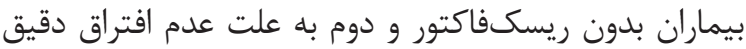
بيماران بدون ريسكفاكتور از بيماران داراى ريسكان عاكاكتور.

حال باتوجلبه اينكه مقاومت اركانيسمهاى ياتوزن نسبت

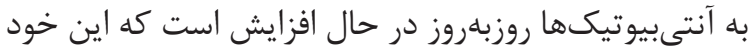

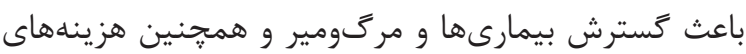

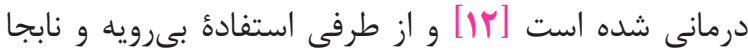

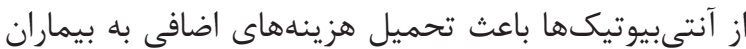

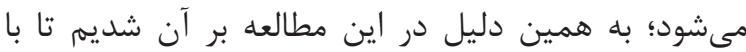

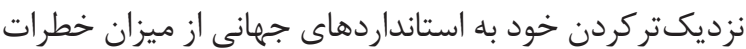

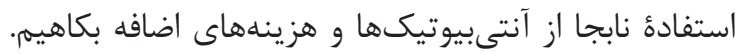

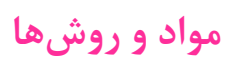

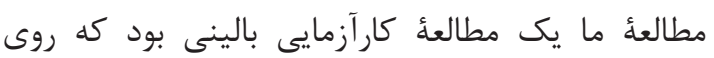

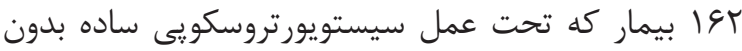

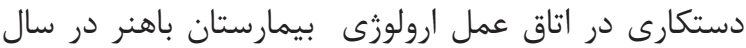

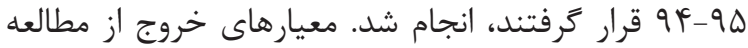

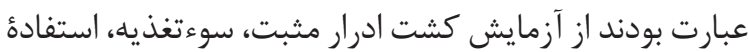




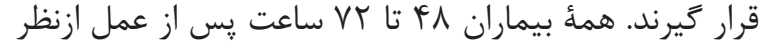

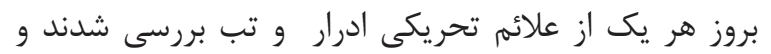

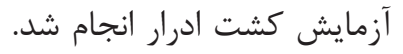

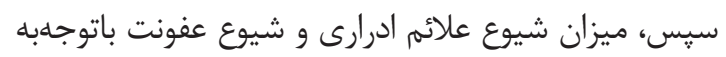

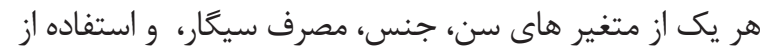

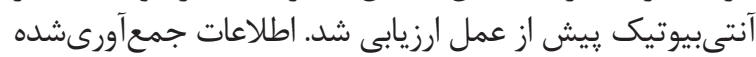

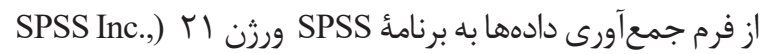

(Chicago, III., USA منتقل شدند و تحليل آمارى شدند.

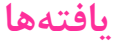

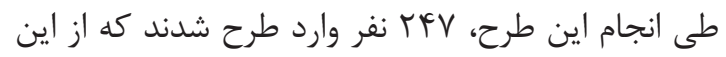

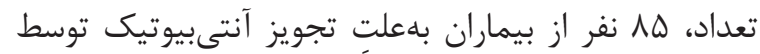

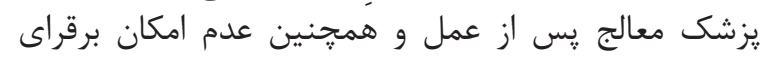

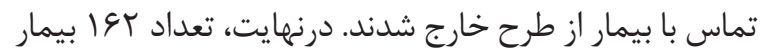

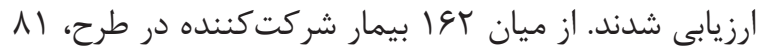

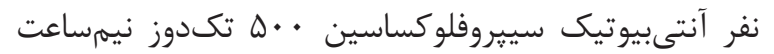

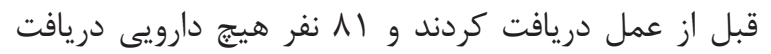

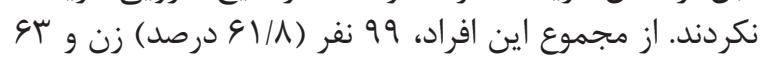

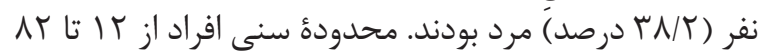
سال با ميانگين سنى (له سال بود بودن )شكل شماره ()).
طولانىمدت از كورتيكواستروئيدها، نقايص سيستمايمنى،

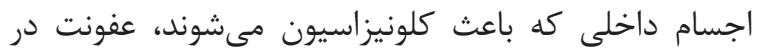

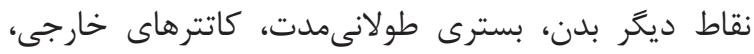

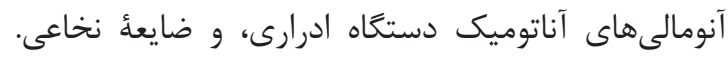

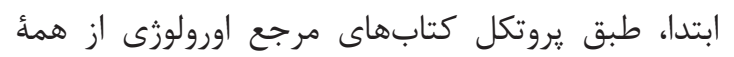

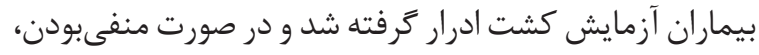

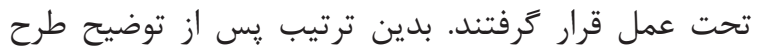

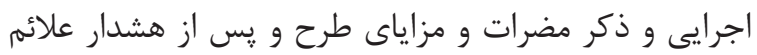

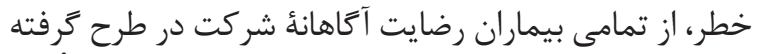

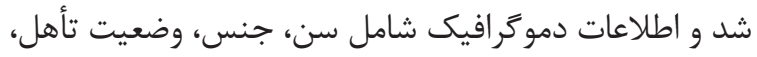

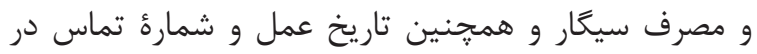

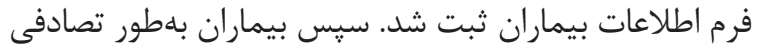

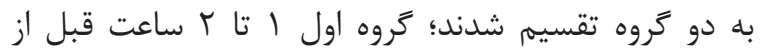

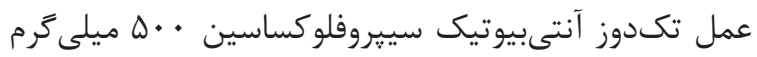

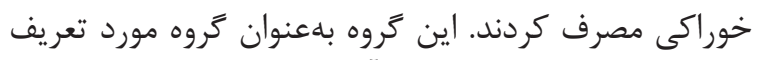

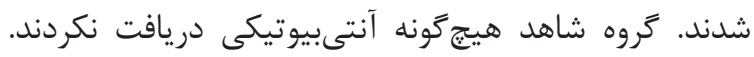

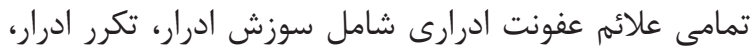

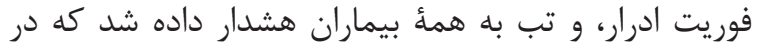

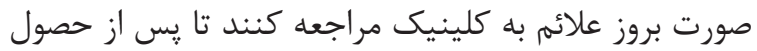
اطمينان از عفونت با آزمايش كشت الينيك ادرار تحت درمان بان مناسب حصول

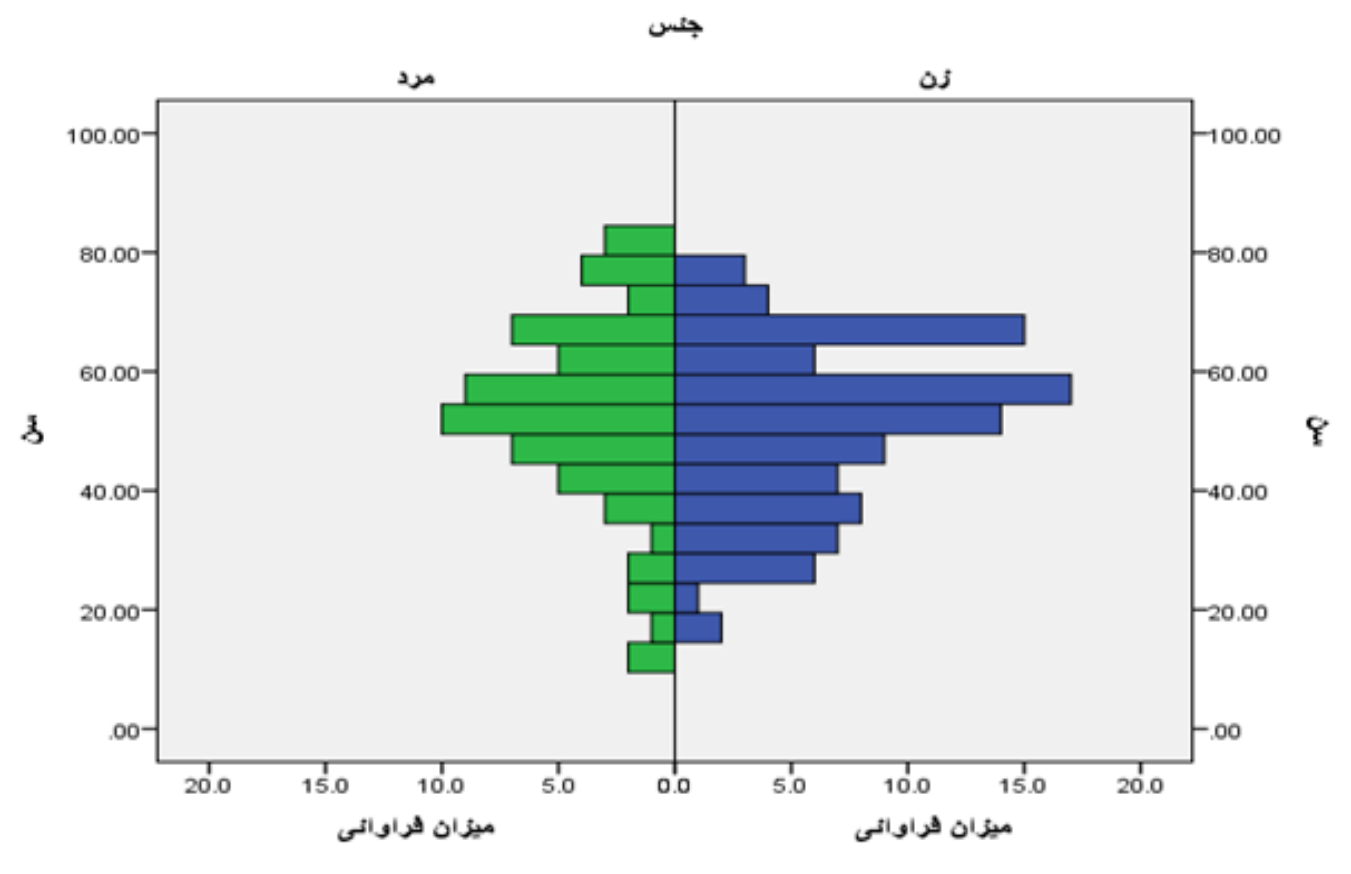

شكل 1. فراوانى براساس سن و جنس

ادرار، سوزش ادرار نيز داشتند. هيج كدام از بيماران دجار تب

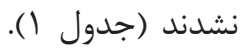

همة افراد تحت بررسى آزمايش كشت ادرار قرار گرفتند؛

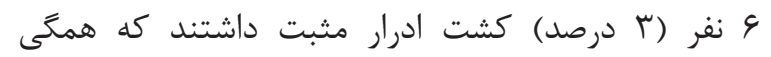

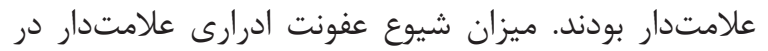

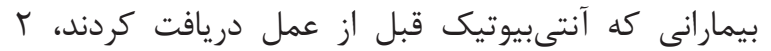

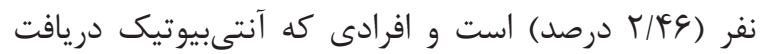

در مطالعه حاضر، از ميان افراد شركت كننده در طرح،

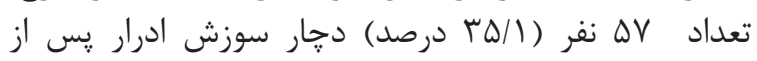

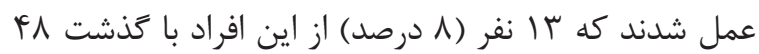

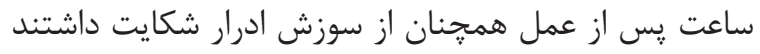

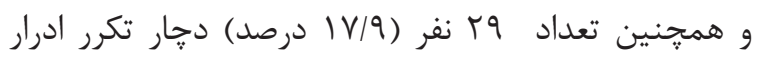

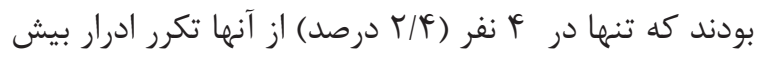

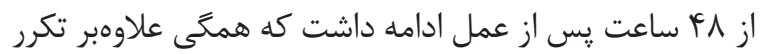




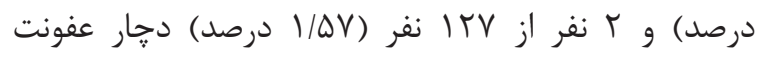

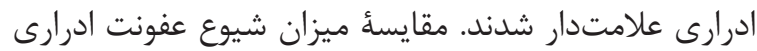

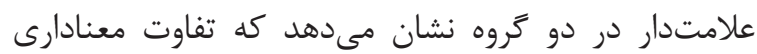

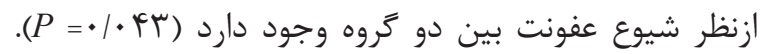

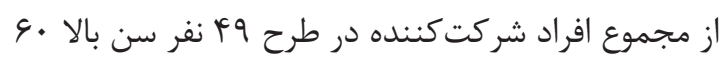

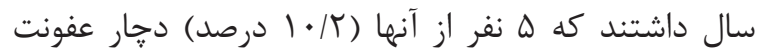

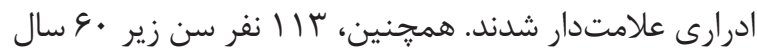

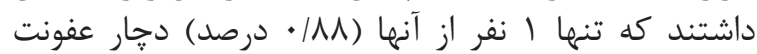

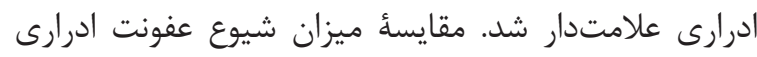

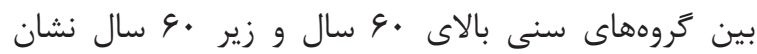

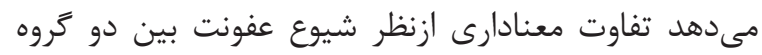

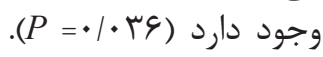

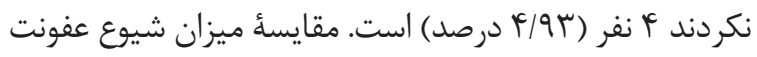

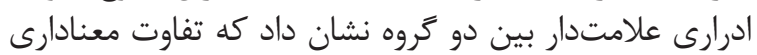

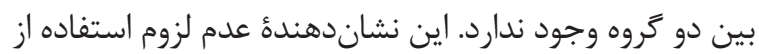

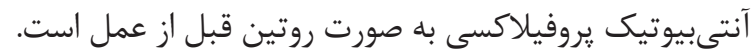

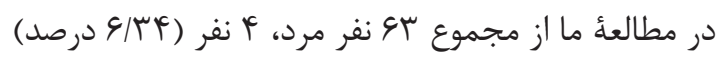

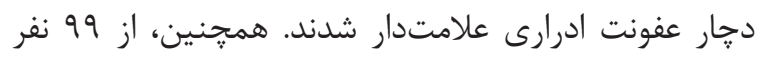

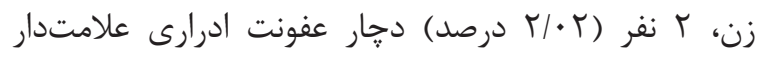

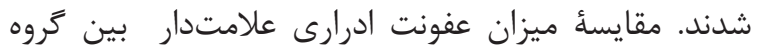

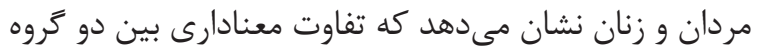

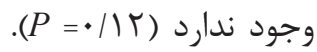

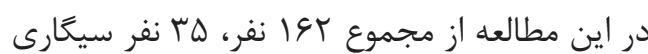

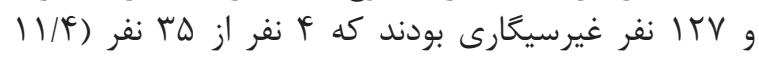

جدول ا ـ شيوع علايم تحريكى در بيماران

\begin{tabular}{|c|c|c|c|}
\hline \multirow{2}{*}{ علائم } & \multicolumn{3}{|c|}{ مدت زمان علائم } \\
\hline & بدون علامت & $<\psi \wedge$ & $\hat{H} \wedge<$ \\
\hline سوزش ادرار & $1 \cdot 0$ & $(\% r \Delta / I) \Delta V$ & r \\
\hline تكرر ادرار & זrו & $(\%) \vee / q) \curlyvee q$ & $(\% r / F) F$ \\
\hline
\end{tabular}

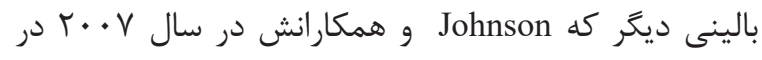

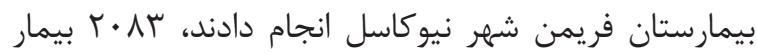

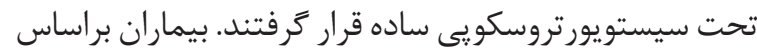

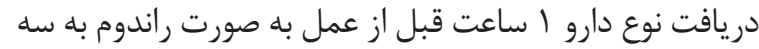

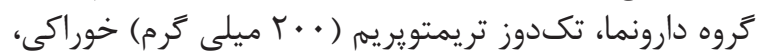

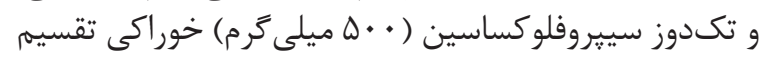

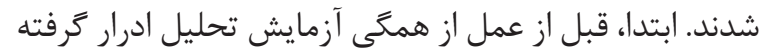

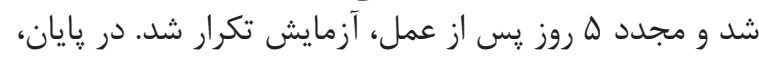

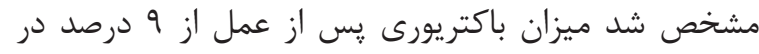

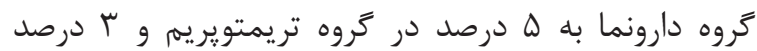

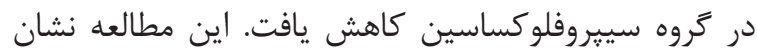

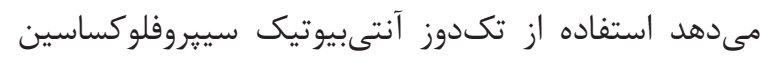

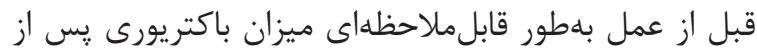

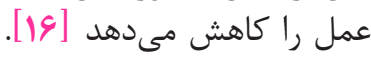
از طرفى، جندين كارآزمايى بالينى ديخر نشان مى ندهد

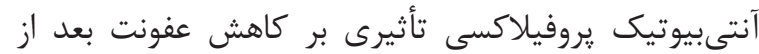

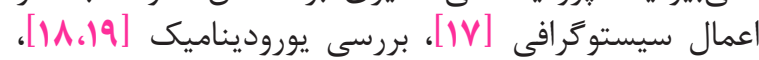

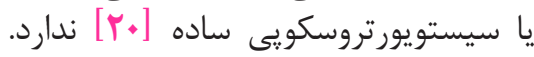
در مطالعهُ Wilson و همكار انش كه در سال ه ه • . ب در تر كيه

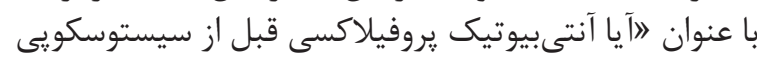

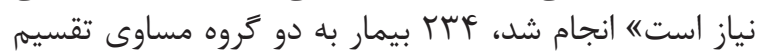

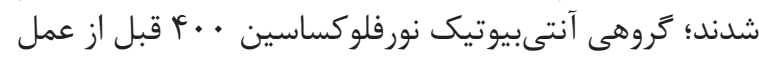

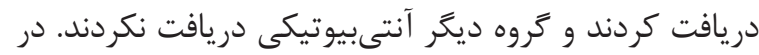

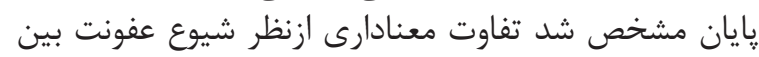

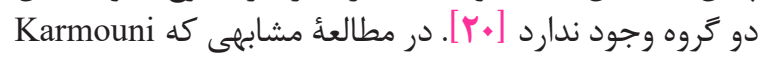

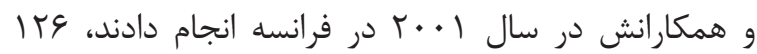

\section{نتيجهَيرى و بحث}

ابتدا، براى درى بهتر عدم وجود اتفاقنظر درمورد نياز و نوع آنتىبيوتيك در جراحى تشخيصى شايع درئ ارولوزى يعنى يعنى

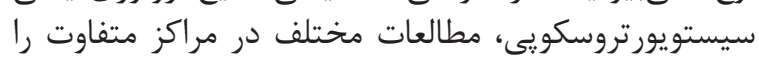
مرور كرديم.

در كارآزمايى بالينى كه توسط Rané و همكارانش در سال

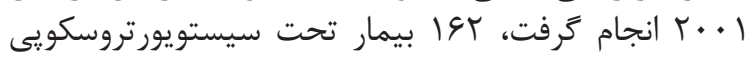

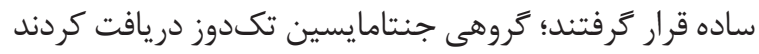

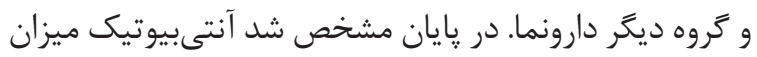

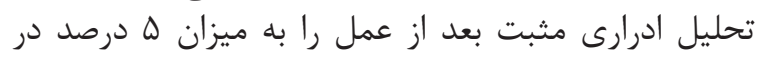

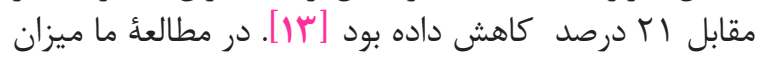

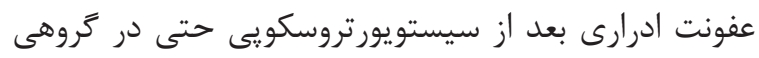

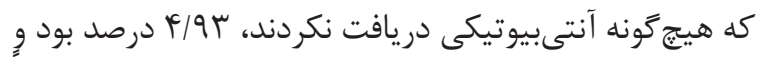

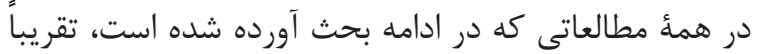

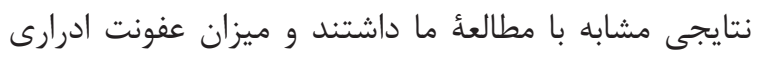

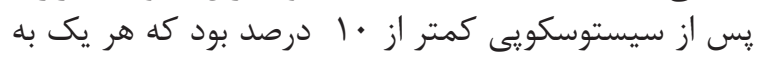
تفصيل در ادامه ذكر خواهد شد. سمتر

در مطالعه Turan و همكارانش كه در بيمارستان كنياى

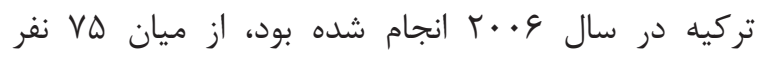

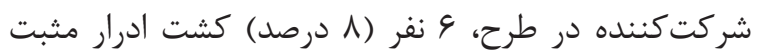

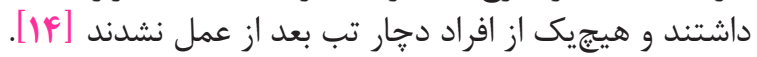

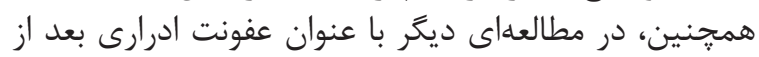

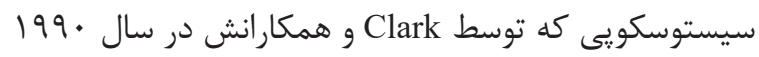

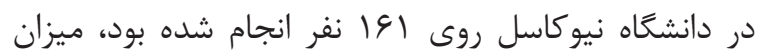

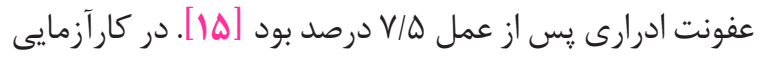




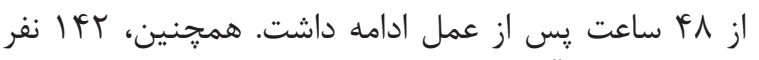

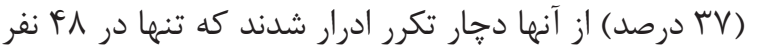

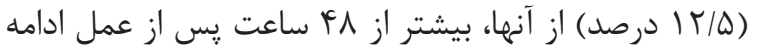

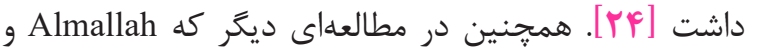
همكارانش با عنوان لاعفونت ادرارى و رضايتمندى دين بيماران

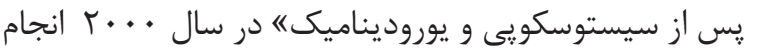

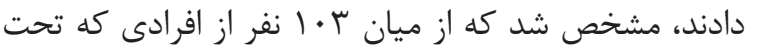

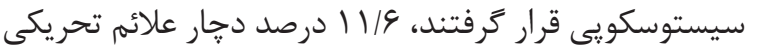

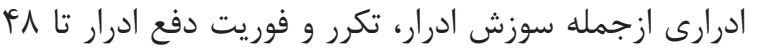

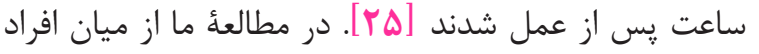

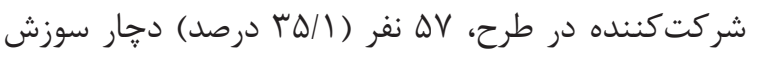

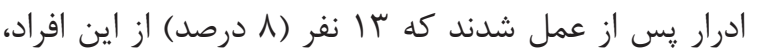

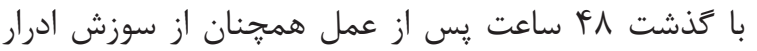

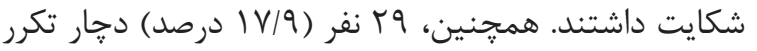

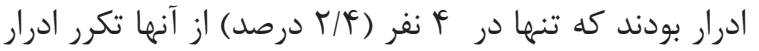

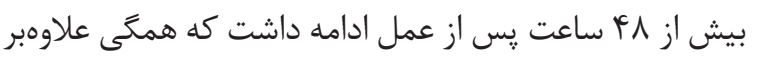
تكرر ادرار، سوزش ادرار نيز داشتند. سئ سئ

براساس مطالعهُ ما نياز به تجويز آنتىبيوتيك يروفيلاكسى سئى

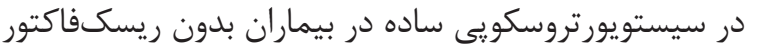

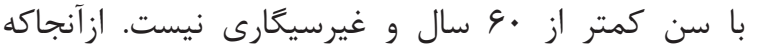

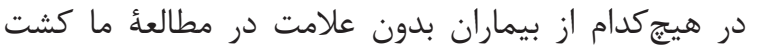

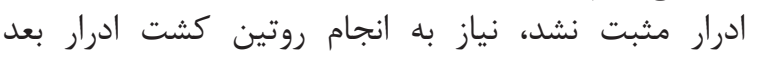

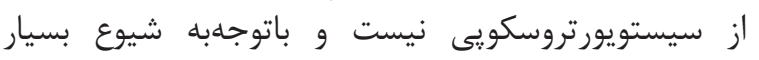

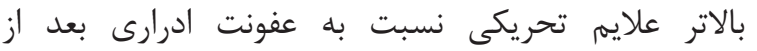

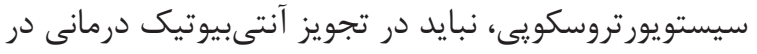

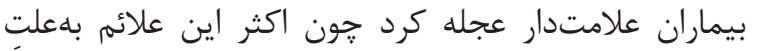

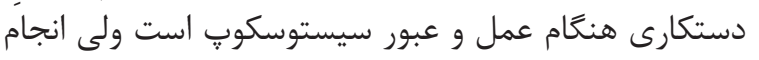
كشت ادرار در بيماران علامتدار توصيه مئ عىشود.

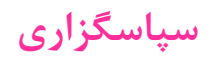

اين مطالعه، نتيجه طرح : يزوهشى مصوب به شماره

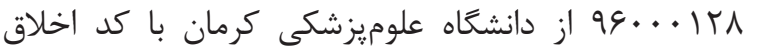
IR.KMU.REC1396.1291 بيمارستان شهيدباهنر كه در اين طرح ما را يارى كردند

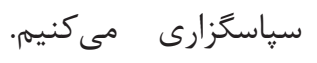
تعارض در منافع بين نويسند
نفر تحت سيستوسكويى قرارگرفتند. تعداد $9 V$ نفر از آنها

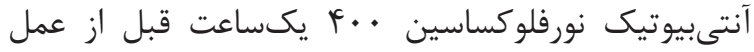

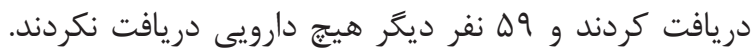

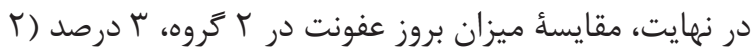

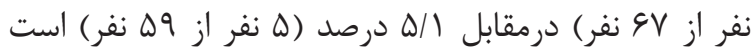

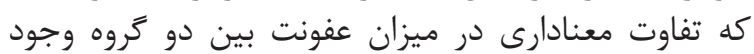
نداشت [r)]

در مطالعهاى ديخر كه Kamil Cam و همكارانش در

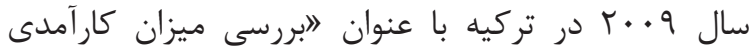

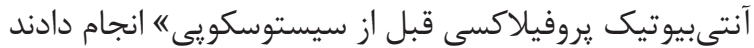

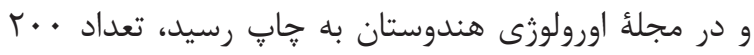

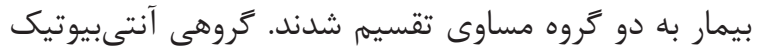

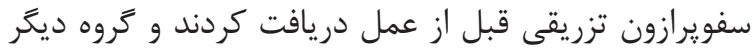

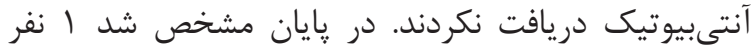

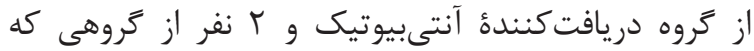

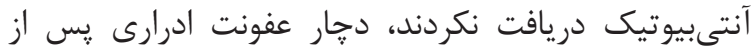

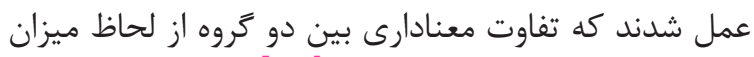

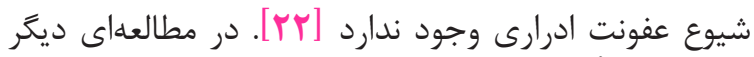

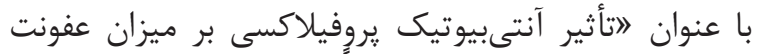

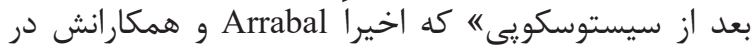

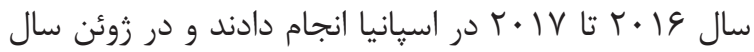

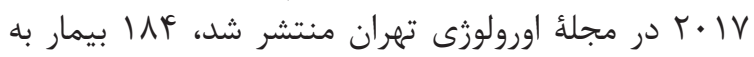

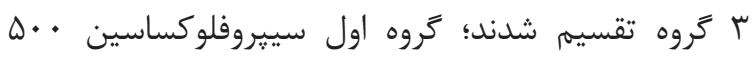

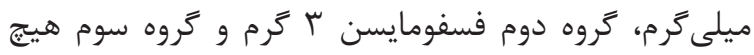

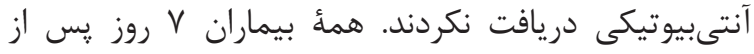

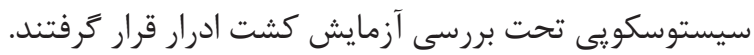

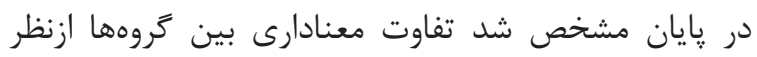

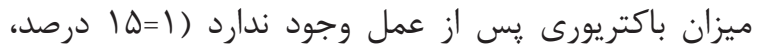
تأثي سا

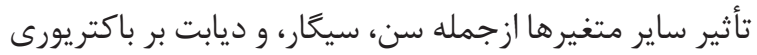

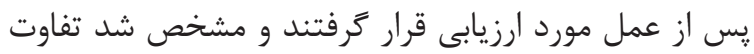

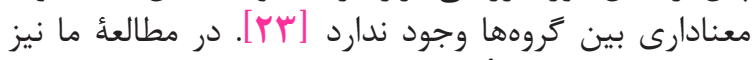

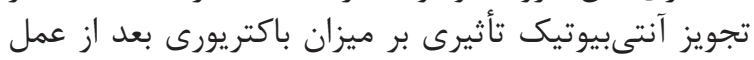

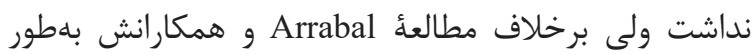

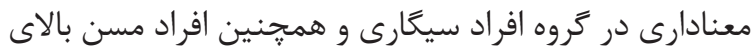

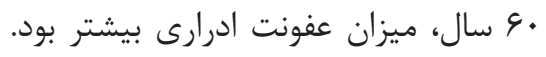

در بررسى مطالعات، نتايج متفاوتى دررابطهبا تأثير

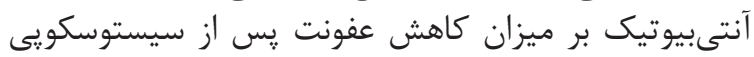

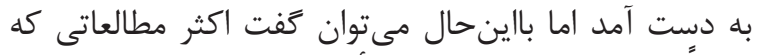

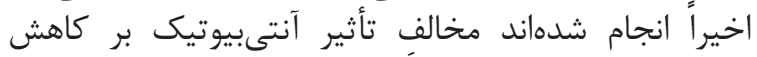

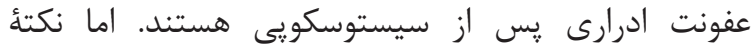

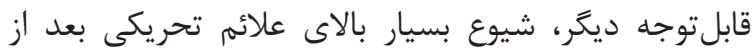

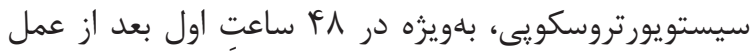

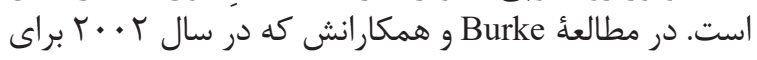

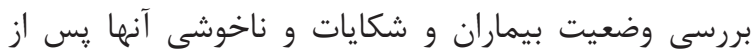

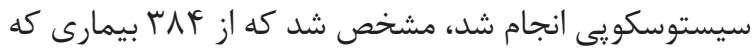

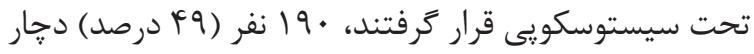

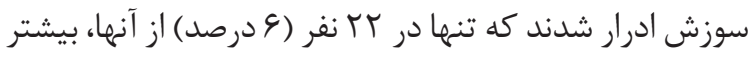




\section{References}

1. Bratzler DW, Houck PM. Workgroup, Antimicrobial prophylaxis for surgery: an advisory statement from the National Surgical Infection Prevention Project. The American Journal of Surgery. 2005. 189(4):395404 [DOI:10.1016/j.amjsurg.2005.01.015] [PMID]

2. Kirkland KB, Briggs JP, Trivette SL, Wilkinson WE, Sexton DJ. The impact of surgical site infections in the 1990s: attributable mortality, excess length of hospitalization, and extra costs. Infect Control Hosp Epidemiol 1999; 20(11):725-30. [DOI:10.1086/501572] [PMID]

3. National Nosocomial Infections Surveillance (NNIS) report, data summary from October1986-April 1996, issued May 1996. A report from the National Nosocomial Infections Surveillance (NNIS) System. Am J Infect Control 1996; 24(5):380-8. [DOI:10.1016/S0196-6553(96)90026-7]

4. Bratzler DW, Houck PM, Richards C, Steele L, Dellinger EP, Fry DE, et al. Use of antimicrobial prophylaxis for major surgery: baseline results from the National SurgicalInfection Prevention Project. Arch Surg 2005; 140(2):174-82. [DOI:10.1001/ archsurg.140.2.174] [PMID]

5. Bold RJ, Mansfield PF, Berge, DH, Pollock RE, Singletary SE, Ames FC et al. Prospective, randomized, double-blind study of prophylactic antimicrobials in axillarylymph node dissection. Am J Surg. 1998; 176(3): 239-43. [DOI:10.1016/S00029610(98)00154-8]

6. Mazza A. Ceftriaxone as short-term antimicrobial prophylaxis in orthopedic surgery: a costbenefitanalysis involving 808 patients. J Chemother. 2000; 12 Suppl 3:29-33. [DOI:10.1080/1120009X.2000.11782305] [PMID]

7. Külling D, Sonnenberg A, Fried $M$ and Bauerfeind P: Cost analysis of antimicrobialprophylaxis for PEG. Gastrointest Endosc. 2000; 51(2):152-6. [DOI:10.1016/ S0016-5107(00)70410-X]
8. Rudge MV, Atallah AN, Peraçoli JC, Tristão Ada R, and Mendonça Neto M. Randomized controlled trial on prevention of postcesarean infection using penicillin and cephalothinin Brazil. Acta Obstet Gynecol Scand2006; 85(8):9458. [DOI:10.1080/00016340600697538] [PMID]

9. Gomez MI, Acosta-Gnass SI, Mosqueda-Barboza L, Basualdo JA. Reduction in surgical antimicrobial prophylaxis expenditure and the rate of surgical site infection by means of a protocol that controls the use of prophylaxis. Infect Control Hosp Epidemiol. 2006; 27(12):1358-65. [DOI:10.1017/ S0195941700075305] [PMID]

10. Campbell MF. Campbell-Walsh Urology. Alan J Wein; 2012.

11. Mete C, Tandoğdu Z, Naber K, Tenke P, Wagenlehner F, Edgar van Oostrum et al. Global Prevalence Study of Infections in Urology Investigators. "Antibiotic prophylaxis in urology departments, 2005-2010." European Urology. 2013; 63(2): 38694. [DOI:10.1016/j.eururo.2012.09.038] [PMID]

12. Shlaes DM, Gerding DN, John JF, et al. Society for Healthcare Epidemiology of America Joint Committee on the Prevention of Antimicrobial Resistance: guidelines for the prevention of antimicrobial resistance in hospitals. Clin Infect Dis1997; 18:27591. [DOI:10.1086/647610] [PMID]

13. Rané A, Cahill D, Saleemi A, Montgomery B and Palfrey E: The issue of prophylactic antimicrobials prior to flexible cystoscopy. Eur Urol 2001; 39(2):212-4. [DOI:10.1159/000052438] [PMID]

14. Turan H, Balci U, Erdinc FS, Tulek N, Germiyanoglu C. Bacteriuria, pyuria and bacteremia frequency following outpatient cystoscopy. Int J Urol. 2006; 13(1):25-8. [DOI:10.1111/j.1442-2042.2006.01219.x] [PMID]

15. Clark KR, Higgs MJ. Urinary infection following out-patient flexible cystoscopy. Br. J. Urol. 1990; 66(5):503-5. [DOI:10.1111/ 
j.1464-410X.1990.tb14997.x] [PMID]

16. Johnson MI, Merrilees D, Robson WA, Lennon T, Masters J, Orr KE et al. Oral ciprofloxacin or trimethoprim reduces bacteriuria after flexible cystoscopy. Br. J. Urol. 2007; 100(4):826-9. [DOI:10.1111/j.1464410X.2007.07093.x] [PMID]

17. Tsugawa M, Monden K, Nasu Y, Kumon $\mathrm{H}$, Ohmori H. Prospective randomizedcomparative study of antimicrobial prophylaxis in urethrocystoscopy and urethrocystography. . Int J Urol 1998; 5(5):441-3. [DOI:10.1111/j.1442-2042.1998.tb00384.x] [PMID]

18. Cundiff GW, McLennan MT, Bent AE. Randomized trial of antimicrobial prophylaxis for combined urodynamics and cystourethroscopy. Obstet Gynecol 1999; 93(5 Pt 1):749-52. [DOI:10.1097/00006250199905000-00022] [PMID]

19. Peschers UM, Kempf V, Jundt K, Autenrieth I, Dimpfl T. Antimicrobial treatment to prevent urinary tract infections after urodynamic evaluation. Int Urogynecol J PelvicFloor Dysfunct 2001; 12: 254-7. [DOI:10.1007/s001920170048] [PMID]

20. Wilson L, Ryan J, Thelning C, Masters J and Tuckey J. Is antimicrobial prophylaxis required for flexible cystoscopy? A truncated randomized double-blind controlled trial. J Endourol 2005; 19 (8):1006-8. [DOI:10.1089/end.2005.19.1006] [PMID]

21. Karmouni T, Bensalah K, Alva A, Patard JJ, Lobel B, Guille F. Role of antibiotic prophylaxis in ambulatory cystoscopy. Prog. Urol. 2001; 11: 1239-41.

22. Cam K, Kayikci A, Erol A. Prospective evaluation of the efficacy of antibiotic prophylaxis before cystoscopy. Ind J Urol. 2009; 25(2):203-206. [DOI:10.4103/09701591.52914] [PMID] [PMCID]

23. Arrabal-Polo MA, Cano-García MC, Arrabal-Martín M, Merino-Salas S. The Effect of Antibiotic Prophylaxis on Post-Operative Infection in Patients Undergone Flexible Cystoscopy. J Res Urol. 2017; 14(3): 3050-3
24. Burke, DM, Shackley DC, O'Reilly PH. The community-based morbidity of flexible cystoscopy. Br. J. Urol. 2002; 89: 347-349. [DOI:10.1046/j.1464-4096.2001.01899.x] [PMID]

25. Almallah YZ, Rennie CD, Stone J, Lancashire MJR. Urinary tract infection and patient satisfaction after flexible cystoscopy and urodynamic evaluation. Urology. 2000; 56(1):37-9. [DOI:10.1016/S00904295(00)00555-0] 